\title{
Even-Odd Effect in Spontaneously Coherent Bilayer Quantum Hall Droplets
}

\author{
K. Park, V. W. Scarola, and S. Das Sarma \\ Condensed Matter Theory Center and Department of Physics, University of Maryland, College Park, MD 20742-4111
}

(October 24, 2018)

\begin{abstract}
Using exact diagonalization in the disc geometry we predict a novel even-odd effect in the Coulomb blockade spectra of vertically coupled double quantum dots under an external magnetic field. The even-odd effect in the tunneling conductance is a direct manifestation of spontaneous interlayer phase coherence, and is similar to the even-odd resonance in the Cooper pair box problem in mesoscopic superconducting grains. Coherent fluctuations in the number of Cooper pairs in superconductors is analogous to the fluctuations in the relative number difference between the two layers in quantum Hall droplets.
\end{abstract}

PACS numbers:

It is now well accepted [1] that a bilayer quantum Hall system may spontaneously develop interaction-induced interlayer phase coherence with an associated Goldstone mode. Such an interlayer coherent state is akin to an excitonic condensate, being qualitatively similar to a neutral superfluid ground state. Pioneering experiments [2] by Eisenstein and collaborators have firmly established the physical reality of such an interlayer coherent phase in high-mobility bilayer GaAs heterostructures around a total Landau level filling factor of unity $\left(\nu_{T}=1\right)$. One of the most spectacular experimental demonstrations of the superfluidity of this spontaneous coherent phase has been the observation [2] of a very sharp interlayer tunneling peak which has been interpreted by some (but not all) as the direct analog of the Josephson effect [1].

Given the considerable significance of the spontaneous quantum Hall interlayer phase coherence as a novel correlation-induced collective phenomenon, it is important to envisage alternative non-trivial properties of the coherent state which have direct analogies to superconducting systems. In this Letter, we theoretically study one such property, namely the precise analogy between bilayer coherent quantum Hall droplets and the "Cooper pair box" problem in small superconducting grains studied in a series of seminal experiments [3] by Nakamura and collaborators. Our theoretical results presented in this paper shows convincingly that bilayer quantum Hall quantum dot systems could spontaneously (i.e. in the absence of any interlayer tunneling) develop interlayer coherence leading to coherent fluctuations in the number of electrons in each dot (with the total number of electrons in the double dot system being fixed), which in turn will give rise to an experimentally observable evenodd effect analogous to the even-odd resonance (and the corresponding Rabi oscillations) reported in the Cooper pair box experiment [3]. Our predicted even-odd effect in the bilayer quantum Hall quantum dot system has the obvious additional exciting prospect of providing a robust quantum two-level system with the interesting potential of serving as a qubit in a novel quantum-Hall-quantum- dot quantum computer architecture [4], which is fundamentally different from electron-spin-based quantum dot qubits currently being studied in the literature [5].

The system of interest is the so-called verticallycoupled double dot system [6] in a strong, external magnetic field so that the double-dot system is effectively equivalent to a finite bilayer quantum Hall droplet. We also assume that the electron number in each dot can be precisely controlled as has been demonstrated. In particular, we consider the system to have an odd number of electrons, which, to be specific, we take to be 7 without any loss of generality (any other small odd number of electrons such as 5, 9, 11, 13, etc. does not make any difference in our analysis). Due to the large capacitive energy associated with interlayer charge imbalance, the ground state of the system (without any external bias voltages and interlayer tunneling) has double degeneracy: the state with 4 (3) electrons in the top (bottom) layer has exactly the same energy as the state with 3 (4) electrons in the top (bottom) layer. We denote these two degenerate ground states as $|4,3\rangle$ and $|3,4\rangle$, respectively.

For a range of magnetic fields the two degenerate ground states, $|4,3\rangle$ and $|3,4\rangle$, are separated from the excitation spectrum by an energy gap. This is so because the Coulomb interaction energy has a cusp at a particular configuration of states which is usually known as the maximum density droplet (MDD) state [7]. It is important to note that the MDD state in our system is the mesoscopic droplet realization of the bulk bilayer coherent state at $\nu_{T}=1$. The above two MDD states, $|4,3\rangle$ and $|3,4\rangle$, compose our two level system for the finite droplet similar to the corresponding Cooper pair box situation.

We begin our quantitative analysis by considering the Hamiltonian for electrons subject to a uniform magnetic field and a parabolic confining potential. In the FockDarwin basis, the many-body Hamiltonian for the bilayer quantum dot system can be written as:

$$
H=H_{0}+\hat{P} V_{\text {Coul }} \hat{P}+H_{t}
$$


where $H_{0}=\frac{1}{2}\left(\sqrt{\omega_{c}^{2}+4 \omega_{0}^{2}}-\omega_{c}\right) \hat{L}_{z}$, with $\hat{L}_{z}$ being the total angular momentum in $z$-direction. Also, $\omega_{c}$ is the cyclotron frequency and $\omega_{0}$ parameterizes the parabolic confining potential. $\hat{P}$ is the lowest Landau level (LLL) projection operator and $V_{\text {Coul }}$ represents the usual Coulomb interaction between electrons:

$$
\frac{V_{\text {Coul }}}{e^{2} / \epsilon a}=\sum_{i<j \in \uparrow} \frac{1}{r_{i j}}+\sum_{k<l \in \downarrow} \frac{1}{r_{k l}}+\sum_{i \in \uparrow, k \in \downarrow} \frac{1}{\sqrt{r_{i k}^{2}+\left(\frac{d}{a}\right)^{2}}},
$$

where $d$ is the interlayer spacing, $\epsilon$ is the GaAs dielectric constant, and $r_{i j}$ is the lateral separation between the $i$-th and $j$-th electron. The natural length unit is the modified magnetic length $a=l_{B}\left(1+4 \omega_{0}^{2} / \omega_{c}^{2}\right)^{-1 / 4}$ which reduces to the planar magnetic length, $l_{B}=\sqrt{\hbar c / e B}$, when the cyclotron energy is much larger than the confining potential energy. In the above we have used a pseudo-spin representation to describe the double layer system: $\uparrow$ and $\downarrow$ distinguish different layers. In general we define the pseudo-spin operator:

$$
\mathbf{S} \equiv \frac{1}{2} \sum_{m} c_{a}^{\dagger}(m) \vec{\sigma}_{a b} c_{b}(m)
$$

where $\hat{S}_{z}$ measures the electron number difference between layers, and $\hat{S}_{x}$ is associated with interlayer tunneling. We take the real spin to be fully polarized either because of the large Zeeman coupling or because of electron-electron repulsion, i.e. Hund's rule.

The tunneling Hamiltonian $H_{t}$ in the Eq.(1) can be written as:

$$
H_{t}=-\frac{t}{2} \sum_{m} c_{a}^{\dagger}(m) \sigma_{a b}^{x} c_{b}(m) \equiv-t \hat{S}_{x}
$$

where $\sigma$ is the usual Pauli matrix, $t$ is the single particle interlayer tunneling gap, and $m$ denotes the LLL angular momentum quantum number. Eq.(4) is valid for general $t$. However, we are interested in the limit of zero interlayer tunneling, i.e. $t /\left(e^{2} / \epsilon a\right) \rightarrow 0$, which is appropriate when considering spontaneous interlayer coherence (note that the $t \rightarrow 0$ limit is not the same as the $t=0$ situation).

We now analyze the Hamiltonian in Eq.(1) via exact diagonalization of finite size systems in the limit of zero tunneling. In this limit, the Hamiltonian is invariant with respect to layer exchange and spatial rotations, i.e. $\left[V_{\text {Coul }}, \hat{S}_{z}\right]=0$ and $\left[V_{\text {Coul }}, \hat{L}_{z}\right]=0$. We may therefore restrict the Hilbert space to specific $S_{z}$ and $M_{z}$ sectors, where $S_{z}$ and $M_{z}$ are the eigenvalues of $\hat{S}_{z}$ and $\hat{L}_{z}$, respectively. We diagonalize the Coulomb interaction in the basis of LLL single-particle eigenstates. [8]. In particular, we focus our attention on the part of the Hilbert space containing the MDD state which occurs at $M_{z}=N(N-1) / 2$, where $N$ is the total number of particles.
Fig.1 shows the eigenenergy spectrum of the Coulomb interaction as a function of $S_{z}$ for a 7 electron system with $d / a=1$ and $M_{z}=21$. Due to the direct electrostatic contribution of the Coulomb interaction, the lowest energy state is obtained for states with the smallest charge imbalance between layers, i.e. $S_{z}=+1 / 2(|4,3\rangle)$ and $-1 / 2(|3,4\rangle)$. In fact, this electrostatic contribution may be viewed as the relative charging energy between layers. As expected, the ground states located at $S_{z}=1 / 2$ and $-1 / 2$ are separated from the lowest energy states of higher $\left|S_{z}\right|$ by the relative charging energy cost:

$$
V_{\text {Coul }}^{\text {direct }}=\frac{\alpha}{N} \hat{S}_{z}^{2}
$$

where we find $\alpha /\left(e^{2} / \epsilon a\right) \simeq-0.18+0.35 d / a$ for $d / a \gtrsim 0.5$. In particular, the lowest energy state of $S_{z}= \pm 1 / 2$ is separated from that of $S_{z}= \pm 3 / 2$ by an energy gap of roughly $0.05 e^{2} / \epsilon a$. Therefore, as far as excitations lower than this charging energy cost are concerned, we can restrict our attention to the Hilbert space of $S_{z}= \pm 1 / 2$. Note that the energy spectra of the $S_{z}=1 / 2$ and $-1 / 2$ states are identical because of reflection symmetry between layers. Also, it is important to remember that the relative charging energy, $V_{\text {Coul }}^{\text {direct }}$, is inversely proportional to the number of electrons. Therefore, for large $N$, mixing between states with different $S_{z}$ becomes appeciable, in which case our two-level system is ill-defined.

Fig.2 shows the energy spectrum in the $S_{z}=1 / 2$ Hilbert space as a function of $M_{z}$ for 7 electrons at $d / a=$ 1. The energy in the graph is the sum of the Coulomb interaction energy and the confining potential energy: $E=V_{\text {Coul }}+\gamma M_{z}$ where $\gamma=\frac{\hbar}{2}\left(\sqrt{\omega_{c}^{2}+4 \omega_{0}^{2}}-\omega_{c}\right)$. By choosing $\gamma=0.1187 e^{2} / \epsilon a$ we obtain the maximum gap. The MDD state is separated from the edge excitation $\left(\Delta M_{z}=+1\right)$ and the internal excitation $\left(\Delta M_{z}=-1\right)$ by roughly $0.05 e^{2} / \epsilon a$ at the interlayer separation $d / a=1$. Also, the $\Delta M_{z}=0$ excitation is shown to have an energy gap roughly equal to $0.07 e^{2} / \epsilon a$ at $d / a=1$. Fig. 3 plots the lowest energy gaps as a function of $d / a$. As seen from the graph, the energy gap is well developed for $d / a \lesssim 1$. We conclude that the MDD state is stabilized in a suitable range of magnetic fields and interlayer distances for small system sizes.

Now that the two degenerate ground states, $\mid S_{z}=$ $+1 / 2\rangle$ and $\left|S_{z}=-1 / 2\right\rangle$, are shown to be well separated from other excitations in the limit of zero tunneling, we can reduce the whole Hilbert space into the Hilbert space composed of only these two states. In this limit, the reduced Hamiltonian is written as:

$$
H_{\text {red }}=-\Delta_{x} \sigma_{x}+\Delta_{z} \sigma_{z},
$$

where $\sigma_{x}$ and $\sigma_{z}$ are the usual Pauli matrices. In the limit of a small single-particle tunneling gap $t, \Delta_{x}=$ $t\left\langle+1 / 2\left|\hat{S}_{x}\right|-1 / 2\right\rangle$. Also, $\Delta_{z}$ is the relative bias voltage between layers. $\Delta_{x}$ is the renormalized tunneling 
gap which is greatly enhanced from the single-particle tunneling gap, $t$, by the Coulomb interaction. In other words, $\Delta_{x} / t$ is the natural order parameter quantifying spontaneous coherence in bilayer quantum Hall systems. The precise definition of spontaneous phase coherence in our quantum dot system is given by:

$$
\lim _{t \rightarrow 0} \frac{\Delta_{x}}{t}=\lim _{t \rightarrow 0}\left\langle+1 / 2\left|\hat{S}_{x}\right|-1 / 2\right\rangle \neq 0 .
$$

Fig. 4 shows $\Delta_{x} / t$ for a 7 particle system as a function of $d / a$. We see that the interaction-induced coherence effect is sizable for $d / a \lesssim 1$. It is important to note that $\Delta_{x}$ increases with system size, more precisely: $\Delta_{x} \simeq$ $\frac{t}{2}(N+1)$ for small $d / a$.

We have established that the bilayer quantum Hall droplet is a natural two level system with intrinsic coherence. Now we predict the even-odd effect in tunneling conductance, which can be used for experimental confirmation of coherence. Tunneling conductance measurements in quantum dot systems in the Coulomb-blockade regime find conductance peaks when the gate voltage $V_{g}$ is tuned so that the total energy of the $N$ electron system becomes identical to that of the $N+1$ electron system. The total energy of the bilayer quantum Hall quantum dot system includes the total charging energy cost, which is given by:

$$
H_{\text {charging }}=\frac{e^{2}}{2 C}\left(N-\frac{C V_{g}}{e}\right)^{2}
$$

where $C$ is the total capacitance of the double dot system.

Fig.5 shows a schematic diagram illustrating the evenodd effect. Fig.5(a) depicts the case with no interlayer coherence due to either a large interlayer separation or an extremely small single-particle tunneling gap. Fig.5(b) shows that, in the low tunneling limit, due to interlayer coherence, the odd $N$ system acquires an energy splitting between the symmetric $\left(\left|\phi_{+}\right\rangle\right)$and antisymmetric $\left(\left|\phi_{-}\right\rangle\right)$superposition of $|+1 / 2\rangle$ and $|-1 / 2\rangle$, which is $\Delta_{x}$. On the other hand, the ground state energy of the even $N$ system decreases by only $2 \tilde{\Delta}_{x}^{2} / E_{c}$ for small $t$, where $\tilde{\Delta}_{x}=t\left\langle 0\left|\hat{S}_{x}\right| 1\right\rangle$, and $E_{c}$ is the energy difference between $\left|S_{z}=0\right\rangle$ and $\left|S_{z}=1\right\rangle$. As a result, the distance between conductance peaks will oscillate between $e^{2} / C+\left(\Delta_{x}-2 \tilde{\Delta}_{x}^{2} / E_{c}\right)$ and $e^{2} / C-\left(\Delta_{x}-2 \tilde{\Delta}_{x}^{2} / E_{c}\right)$ as a function of $e V_{g}$. A large tunneling gap will eventually destroy the even-odd effect because all electrons will occupy the interlayer-symmetric state. This will mix states with different $S_{z}$, thereby destroying our two-level system. Both large and zero tunneling will therefore lead to evenly spaced Coulomb blockade peaks whereas the even-odd effect will show up for weak (but finite) tunneling.

We emphasize that our predicted even-odd effect is the precise quantum Hall analog of the Josephson effect in the Cooper pair box problem. It is important to note that the number of Cooper pairs in superconductors can be formally mapped to $S_{z}$ in our coherent bilayer quantum Hall system. Therefore, the coherent linear combination of superconducting states with different numbers of Cooper pairs ,which leads to the Josephson effect, is precisely analogous to the linear combination of bilayer quantum Hall states with different $S_{z}$, which is the origin of our even-odd effect.

In conclusion, we have proposed an even-odd effect in tunneling conductance through veritcally coupled double quantum dots in the quantum Hall regime, which is the direct analog of the Josephson effect in mesoscopic superconducting grains.

This work is supported by ARDA and LPS.

[1] B. G. Levi, Phys. Today 54 (5), 14 (2001); J. P. Eisenstein, Phys. World 14 (6), 30 (2001).

[2] I. B. Spielman et al., Phys. Rev. Lett. 84, 5808 (2000); I. B. Spielman et al., Phys. Rev. Lett. 87, 36803 (2001); M. Kellogg et al., Phys. Rev. Lett. 88, 126804 (2002).

[3] Y. Nakamura, C. D. Chen, and J. S. Tasi, Phys. Rev. Lett. 79, 2328 (1997); Y. Nakamura, Yu. A. Pashkin, and J. S. Tsai, Nature (London) 398, 786 (1999); Y. Nakamura, Yu. A. Pashkin, T. Yamamoto, and J. S. Tsai, Phys. Rev. Lett. 88, 047901 (2002).

[4] The possibility of using bilayer quantum dot quantum Hall states as quantum bits has very recently been considered by S. R. Yang et al., Phys. Rev. B 66, 153302 (2002). Our work differs significantly from Yang et al. in the sense that we have considered spontaneous interlayer coherence directly in the limit of zero tunneling (whereas Yang et al. considered the situation with explicit finite tunneling in an external gate voltage). The even-odd effect, the main prediction of our work, was not considered by Yang et al.

[5] X. D. Hu and S. Das Sarma, cond-mat/0211358.

[6] D. G. Austing et al., Physica E 10, 112 (2001).

[7] A. H. MacDonald, S. R. Eric Yang, and M. D. Johnson, Aust. J. Phys. 46, 345 (1993).

[8] S. M. Girvin and T. Jach, Phys. Rev. B 29, 5617 (1984); R. K. Kamilla and J. K. Jain, Phys. Rev. B 52, 2798 (1995). 


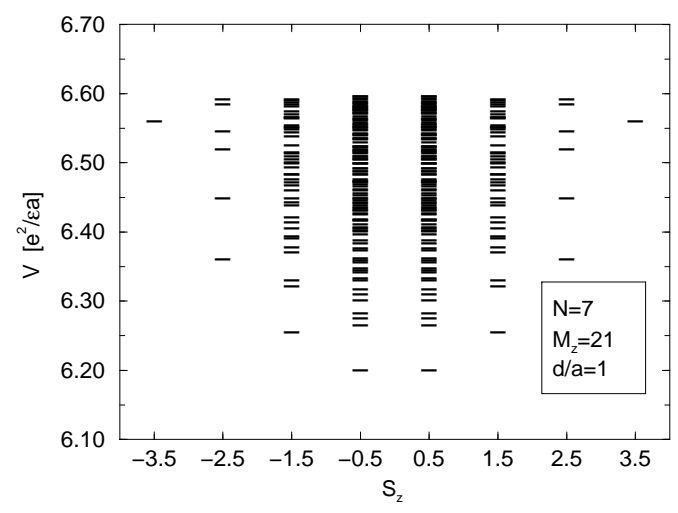

FIG. 1. Coulomb interaction energy as a function of $S_{z}$ which is half the relative electron number difference between different layers. The layer separation is chosen to be $d / a=1$ where we define $a=l_{B}\left(1+4 \omega_{0}^{2} / \omega_{c}^{2}\right)^{-1 / 4}$, the magnetic length $l_{B}=\sqrt{\hbar c / e B}$, and the cyclotron frequency $\omega_{c}=e B / m^{*} c$. $\omega_{0}$ is the frequency of the confining potential. The state with angular momentum $M_{z}=21$, is the maximum density droplet state for $N=7$.

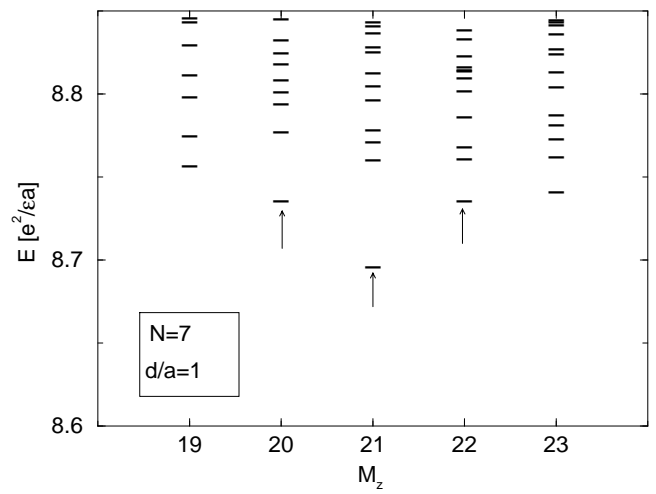

FIG. 2. Energy spectrum as a function of total angular momentum, $M_{z}$, in the Hilbert space of $S_{z}=1 / 2$. Note that the energy in the graph is a sum of the Coulomb interaction energy and the confining potential energy: $E=V_{\text {Coul }}+\gamma M_{z}$ where $\gamma=\frac{\hbar}{2}\left(\sqrt{\omega_{c}^{2}+4 \omega_{0}^{2}}-\omega_{c}\right)$. In the graph, we have chosen $\gamma=0.1187 e^{2} / \epsilon a$ which gives us the largest possible gap. The arrows indicate the three lowest energies.

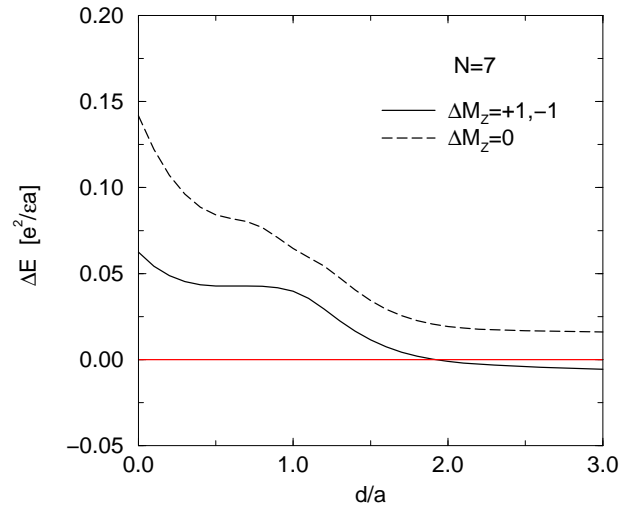

FIG. 3. The lowest three energy gaps as a function of interlayer separation $d / a$. The lowest three excitations are categorized as follows: (i) $\Delta M_{z}=+1$ (the edge excitation), (ii) $\Delta M_{z}=0$ and (iii) $\Delta M_{z}=-1$ (the internal excitation). The energy gap is given by: $\Delta E=\Delta V_{\text {Coul }}+\gamma \Delta M_{Z}$ where $\gamma$ is defined as in Fig. 2.

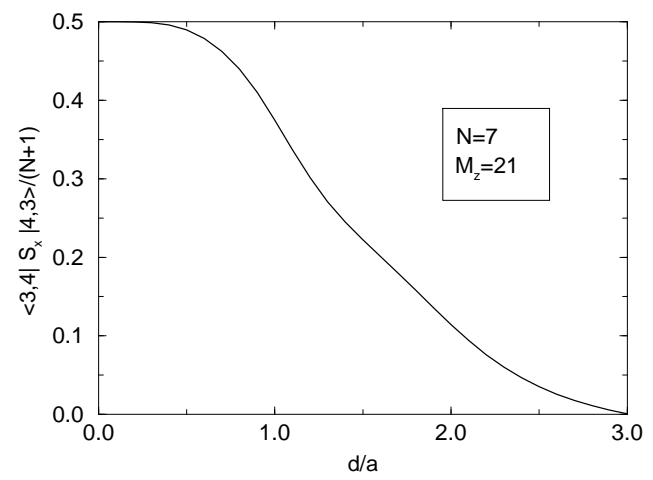

FIG. 4. Interlayer coherence in the limit of zero tunneling as a function of interlayer separation $d / a .|4,3\rangle$ represents the lowest energy state with 4 (3) electrons in the top (bottom) layer for a seven electron system, which can be alternatively denoted by $\left|S_{z}=+1 / 2\right\rangle$. $|3,4\rangle\left(\left|S_{z}=-1 / 2\right\rangle\right)$ is similarly defined. 


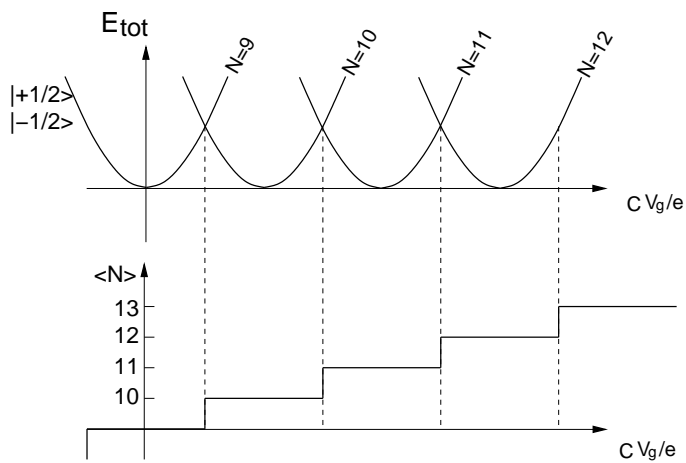

(a)

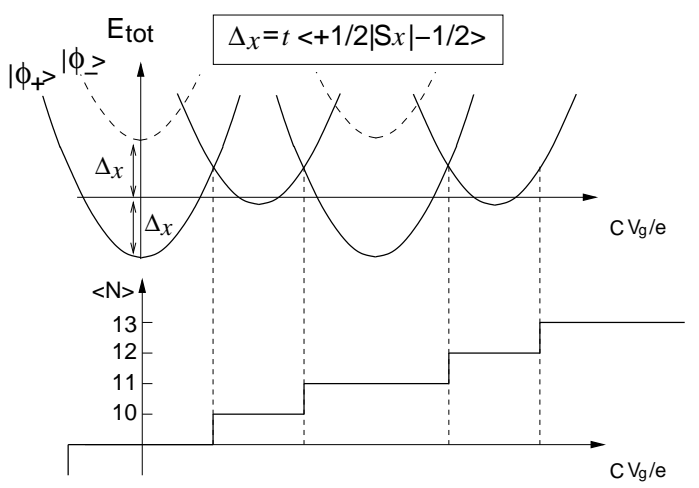

(b)

FIG. 5. Schematic diagram illustrating the even-odd effect in bilayer quantum Hall dots. The total energy including the charging energy is plotted as a function of $C V_{g} / e$ where $V_{g}$ is the gate voltage and $C$ is the capacitance of the whole bilayer system. $\langle N\rangle$ is the average number of electrons inside double quantum dots. Fig.5(a) depicts the situation where there is no interlayer coherence. $|+1 / 2\rangle(|-1 / 2\rangle)$ represents the degenerate set of low energy states with $S_{z}=1 / 2(-1 / 2)$. It is shown in Fig.5(b) that, due to the interlayer coherence, the odd $N$ system acquires an energy splitting between the symmetric $\left(\left|\phi_{+}\right\rangle\right)$and antisymmetric $\left(\left|\phi_{-}\right\rangle\right)$superposition of $|+1 / 2\rangle$ and $|-1 / 2\rangle$, which is $\Delta_{x}$. 